\title{
A posteriori error estimation for standard finite element analysis
}

\author{
Pedro Díez, Juan José Egozcue, Antonio Huerta* \\ Departamento de Matemática Aplicada III, E.T.S. de Ingenieros de Caminos, Universitat Politècnica de Catalunya, Campus Nord C-2, \\ E-08034 Barcelona, Spain
}

\begin{abstract}
A new residual type estimator based on projections of the error on subspaces of locally supported functions is presented. The estimator is defined by a standard element by element refinement. First, an approximation of the energy norm of the error is obtained solving local problems with homogeneous Dirichlet boundary conditions. A later enrichment of the estimation is performed by adding the contributions of projections on a new family of subspaces. This estimate is a lower bound of the measure of the actual error. The estimator does not need to approximate local boundary conditions for the error equation. Therefore, computation of flux jumps is not necessary. Moreover, the estimator can be applied in mixed meshes containing elements of different shapes and its implementation in a standard finite element code is straightforward. The presented results show the effectiveness of the estimator approximating both the distribution and the global measure of the error, as well as its usefulness in adaptive procedures.
\end{abstract}

\section{Introduction}

It is now widely accepted that adaptive procedures are necessary for any practical Finite-Element (FE) computations [1]. Error estimation is a key feature of an adaptive procedure. An error estimator provides information about the global quality of the solution and the distribution of the error in the domain. The global estimate assesses admissibility of the approximate solution. If it is not admissible, a new mesh must be created following a remeshing criterion based on the error distribution. Elements are concentrated where the approximate solution is less accurate. The goal is a new solution with a uniform error distribution and a specified global error.

A priori error estimates are the main tool for the theoretical study of the FE Method but they cannot provide practical results [2]. In order to obtain numerical approximations of the actual error, it is necessary to use a posteriori error estimators. These estimators may be classified into two families: flux projection and residual type estimators.

Flux projection estimators are derived from the original Zienkiewicz-Zhu estimator [3]. A modified version of this estimator, based on the superconvergent patch recovery, has been introduced in [4]. The main idea of such estimators is to approximate the actual error by the difference of a smooth recovered solution and the FE solution. These estimators have a good performance if the recovered solution improves the FE approximation. The recovery techniques are based on superconvergence results. The points where the solution has an order of convergence higher than expected are taken as sample points. Usually, superconvergence is concerned with the values of the fluxes: superconvergent points do not hold more accurate values of the main variable but only of its derivatives. Therefore, flux projection estimators can only be employed if the error is measured using norms that can be expressed in terms of the flux. This applies in the elastic mechanical model using the energy norm.

* Corresponding author. E mail: huerta@etseccpb.upc.es 
Although these estimators have been found to be robust, see [2] and [5], the superconvergence properties are only proved in very few cases [6]. Actually, the choice of the sample points used in the recovery technique does not alter the performance of the estimator [2]. Superconvergence is not observed in meshes mixing different element types, therefore flux projection estimates should not be used with such meshes. Moreover, the recovered solution usually shows a poor quality near the boundaries. Nevertheless, flux projection estimators are the most popular and they have been implemented in many commercial FE codes because of their easy implementation and practical efficiency. Flux projection estimates can be computed using only the existing framework of any classical FE code.

Residual type estimators were first introduced by Babuška and Rheinboldt in [7]. The residual of the FE solution is used as a source term in local boundary value problems associated with the error. These estimators are defined by two factors: the selection of the local interpolation space (usually a bubble space for a $p$-refined reference solution) and the approximation of the boundary conditions by splitting the flux jump across the edges of the elements. Indeed, flux jump can be thought as a weak residual. These kind of estimators have a solid theoretical background but their results have been found to be less robust than flux-projection estimates [2]. The biggest part of the computational cost of these estimators is taken by the flux jump computation and the flux splitting procedure. The implementation of these estimators is not widespread. This is probably because they need computation of specific values which are useless for the general FE analysis.

Some estimators are difficult to classify into one of these groups. The estimators introduced by Ladevèze in [8] and Ohtsubo and Kitamura in [9] build a regularized flux field and therefore they can be put into the first class but they solve local problems associated with the residual and, consequently, they can also be seen as residual type estimators (see [2]). These estimators present the usual disadvantages of the second family.

The estimators defined by Erikkson and Johnson in [10] cannot be classified into one of the above groups.
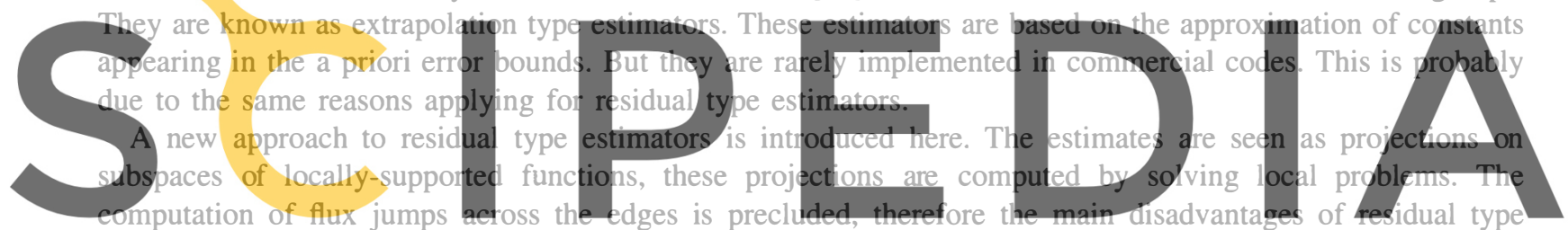

estimators do not hold. Moreover, the estimator can be applied in any kind of meshes, even if different element

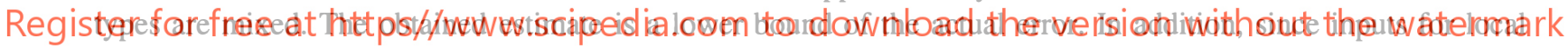

problems are the same as those for the standard problem, the definition and the computation of the estimator are easily integrated in a finite element code.

The remainder of the paper is organized as follows: Section 2 presents the model problem and notation. Section 3 is devoted to error projection computation and some local properties of projections. In Section 4 an interior elementwise estimator is defined. Section 5 deals with the problem of improving the estimation by defining new projection spaces in a convenient way. In Section 6 we show some examples and demonstrate the effectiveness of the estimator.

\section{Model problem}

Let us consider an elliptic problem over a bounded domain $\Omega \subset \mathbb{R}^{2}$ with a piecewise smooth boundary $\Gamma$ divided into two parts $\Gamma_{n}$ and $\Gamma_{d}$. The boundary value problem is defined in strong form as

$$
\begin{aligned}
& -\nabla \cdot(A \nabla u)+b u=f \text { in } \Omega, \\
& u=0 \text { on } \Gamma_{d}
\end{aligned}
$$

and

$$
(A \nabla u) \cdot n=g_{n} \text { on } \Gamma_{n},
$$

where $u$ is defined on $\Omega$ and needs to be approximated. The Dirichlet boundary condition (1b) is taken homogeneous, but, due to the linearity of (1a) this is not a loss of generality, the examples will present non-homogeneous Dirichlet boundary conditions. In order to simplify the presentation, a scalar case is 
considered in the theoretical development of the estimator. However, the extension to vectorial problems is straightforward and plane mechanical examples with two degrees of freedom are shown in Section 6.

In order to study the weak form of the problem, equivalent to the strong form (1), several definitions are needed. Let $H^{\prime}(\Omega)$ denote the usual Sobolev space of functions with square-integrable derivatives and let $H_{\Gamma_{d}}^{3}(\Omega)$ be such that $H_{\Gamma_{d}}^{1}(\Omega)=\left\{w \in H^{1}(\Omega) \mid w=0\right.$ on $\Gamma_{d}$. The solution $u$ of $(1)$ is also the weak solution of the following integral problem: find $u$ in $H_{\Gamma_{d}}^{1}(\Omega)$ such that

$$
a(u, v)=l(v)
$$

for all $v$ in $H_{\Gamma_{d}}^{1}(\Omega)$, where $a(\cdot, \cdot)$ and $l(\cdot)$ are given by

$$
a(u, v):=\int_{\Omega}(\nabla v \cdot A \nabla u+b u v) \mathrm{d} \Omega
$$

and

$$
l(v):=\int_{\Omega} v f \mathrm{~d} \Omega+\int_{\Gamma_{n}} v g_{n} \mathrm{~d} \Gamma .
$$

The Galerkin FE solution $u_{h}$ belongs to a finite dimensional subspace $\mathscr{V}_{h}$ of $H_{r_{d}}^{1}(\Omega)$ and verifies (2) for all $v$ in $\mathscr{V}_{h}$. The goal of error estimation is to approximate a measure of the error $e$, defined as $e:=u-u_{h}$.

If $A$ is a symmetric positive definite matrix and $b$ is non-negative, see Eq. (la), then the bilinear form $a(\cdot, \cdot)$ is also symmetric positive definite and, therefore, it is a scalar product. The norm $\|\cdot\|$ induced by $a(\cdot, \cdot)$ is called the

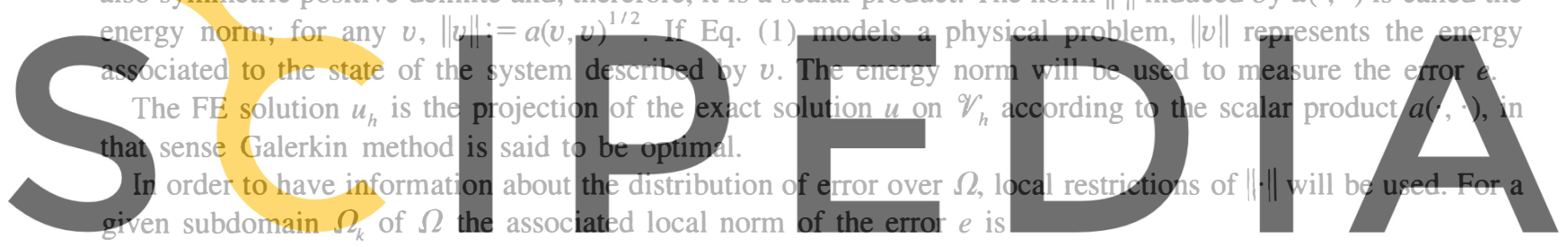

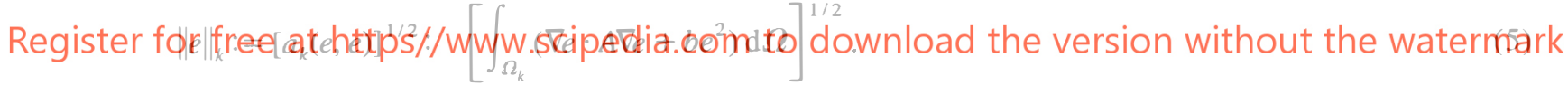

In the following sections, subdomains $\Omega_{k}$ are chosen as the elements of the mesh which defines the approximating space $\mathscr{V}_{h}$ (computational mesh).

\section{Error projections}

An error estimate should provide local information of the error in order to describe its spatial distribution. The computation of an error estimate must also be local; that is the computation of a local restriction of the error in a subdomain $\Omega_{k}$ only uses information in it and, eventually, in its neighborhood. Otherwise, error estimation becomes too expensive and it could be cheaper to use a refined mesh in order to obtain a more accurate solution. The local estimator presented in the following section is made independent of the neighbor elements by forcing the approximation to $e$ to vanish on the boundary. Then, in Section 5 the estimator is improved, precluding the imposition of this artificial condition. before entering in the detailed definition of a local estimator, we discuss error projections which are the rationale of the proposed estimator.

Since the weak equation (2) is well posed and the form $a(\cdot, \cdot)$ is assumed to be linear on the first argument, the error $e$ is the only element of $H_{\Gamma_{d}}^{1}(\Omega)$ verifying

$$
a(e, v)=\tilde{l}(v):=l(v)-a\left(u_{h}, v\right)
$$

for all $v$ in $H_{\Gamma_{d}}^{1}(\Omega)$. Eq. (6) is the weak global equation for the error. Thus, although the error is not known, its scalar product with any given function $v$ may be computed. That is, the projection of $e$ on any subspace of $H_{\Gamma_{d}}^{1}(\Omega)$ can be evaluated. 
Let $V_{k}$ be a subspace of $H_{\Gamma_{d}}^{1}(\Omega)$ of functions having their support in $\Omega_{k}$ : they vanish elsewhere and, consequently, on the boundary of $\Omega_{k}\left(V_{k}\right.$ is included in $\left.H_{0}^{1}\left(\Omega_{k}\right)\right)$. The subspace $V_{k}$ is generated by a basis of interpolation functions $\mathscr{B}=\left\{N_{1}, \ldots, N_{n}\right\}$. Reference to index $k$ in the notation of the elements of $\mathscr{B}$ is omitted when it is not necessary. The projection of $e$ on $V_{k}$ is denoted by $\varepsilon_{k}$, the element $\varepsilon_{k}$ of $V_{k}$ is represented by the column vector $[\varepsilon]_{\mathscr{B}}$ of its components on $\mathscr{B}$, the scalar product $a(\cdot, \cdot)$ is represented by matrix $\boldsymbol{K}$ with generic term $K_{i j}=a\left(N_{i}, N_{j}\right)(i, j=1, \ldots, n)$ and the linear form $\tilde{l}(\cdot)$ is represented by vector $[\tilde{l}]$ with generic term $[\tilde{l}]_{j} \quad l\left(N_{j}\right)$. Finally, the projection $\varepsilon_{k}$ is obtained by solving the linear system of equations

$$
\boldsymbol{K}[\varepsilon]_{\mathscr{B}} \quad[\tilde{l}] .
$$

Then, the energy norm of $\varepsilon_{k}$ can be computed in a straightforward manner by the expression

$$
\left\|\varepsilon_{k}\right\|^{2}=[\varepsilon]_{\mathscr{B}}^{\mathrm{T}} \boldsymbol{K}[\varepsilon]_{\mathscr{R}}=[\varepsilon]_{\mathscr{B}}^{\mathrm{T}}[\tilde{l}] \text {. }
$$

The set of subspaces $V_{k}$ is orthogonal with respect to $a(\cdot, \cdot)$ because all subdomains $\Omega_{k}$ (the finite elements) have intersection with measure zero. Bessel's inequality applies and the norms of projections $\varepsilon_{k}$ verify

$$
\sum_{k=1}^{m}\left\|\varepsilon_{k}\right\|^{2}=\sum_{k=1}^{m} a\left(\varepsilon_{k}, \varepsilon_{k}\right) \leqslant a(e, e)=\|e\|^{2},
$$

where $m$ stands for the number of elements in the mesh.

The bound given by (9) holds even locally. Indeed, as $\varepsilon_{k}$ is the projection of $e$ on $V_{k}$ according to the global form $a(\cdot, \cdot)$, the difference $e \quad \varepsilon_{k}$ is orthogonal to $\varepsilon_{k}$, i.e. $a\left(e-\varepsilon_{k}, \varepsilon_{k}\right)=0$. Moreover, this orthogonality condition also stands for the local form $a_{k}(\cdot, \cdot)$, i.e. $a_{k}\left(e \quad \varepsilon_{k}, \varepsilon_{k}\right)=0$, because the support of $\varepsilon_{k}$ is included in
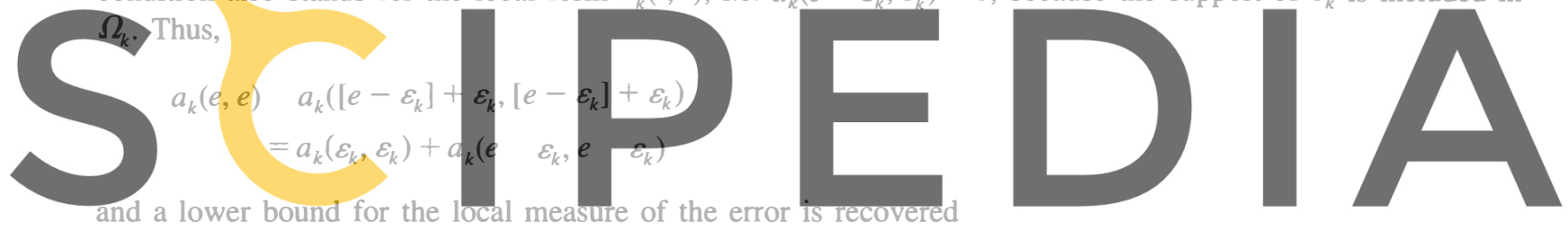

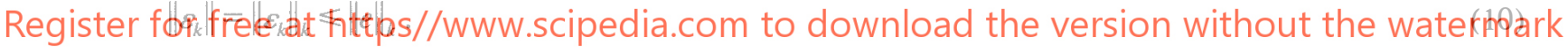

Accordingly, we deduce that a family of subspaces $V_{k}$ of $H_{\Gamma_{d}}^{1}(\Omega)$, defined over disjoint subdomains $\Omega_{k}$ (or with null intersection), allows us to compute local projections $\varepsilon_{k}$ such that:

- the norm of each $\varepsilon_{k}$ is a lower bound of the local norm of the true error, see Eq. (10), and can be taken as a local error estimator,

- defining $\varepsilon$ as the sum of the $\varepsilon_{k}$,

$$
\varepsilon:=\sum_{k=1}^{m} \varepsilon_{k}
$$

the global norm of $\varepsilon$ is easily computed from the local projections and gets a lower bound of the global measure of the error $\|e\|$

$$
\|\varepsilon\|^{2}=\sum_{k=1}^{m}\left\|\varepsilon_{k}\right\|^{2} \leqslant\|e\|^{2}
$$

- both global and local approximations, $\varepsilon$ and the $\varepsilon_{k}$ 's, would be more accurate as the space $V$, generated by the $V_{k}$ 's, $V:=V_{1} \oplus V_{2} \oplus \cdots \oplus V_{m}$, is closer to $H_{\Gamma_{d}}^{1}(\Omega)$ (asymptotic behavior of Bessel inequality (9) is given by Parseval's equality). The global approximation $\varepsilon$ is the projection of $e$ on $V$.

The functions of each subspace $V_{k}$ vanish on the boundary of $\Omega_{k}$. Then, the global approximation $\varepsilon$ associated with a family of $V_{k}$ 's, defined from a partition of $\Omega$ into subdomains $\Omega_{k}$, takes zero values at the points lying on the boundary of the $\Omega_{k}$ 's. Those are called hidden points of the global approximation. In fact, we are artificially forcing $\varepsilon$ to be zero at these points. The space $V$ will never approximate enough $H_{\Gamma_{d}}^{1}(\Omega)$ if many points of the domain are hidden. In the following sections we define new families of subspaces related to other partitions of $\Omega$ in order to reduce these hidden points. 


\section{Definition of a local estimator. Interior residuals}

The definition of the local subspaces $V_{k}$ characterizes the family of functions $\varepsilon_{k}$. The $\varepsilon_{k}$ 's are taken as approximations of the local error. That is, approximations to the error inside each element $\Omega_{k}$ where the subspace $V_{k}$ is defined. Local subspaces for residual type estimators have been typically defined following a $p$-refinement [11-13] or an $h$-refinement strategy [14]. The present estimator can follow both approaches. For the sake of simplicity we only show an $h$-refinement approach.

The interpolation functions $N_{i}$ generating $V_{k}$ are defined by a discretization of the element $\Omega_{k}$. We call these meshes elementary submeshes. In order to make computations systematic and to have a simple implementation, we describe an elementary submesh over a reference element. Generic elementary submeshes are built applying the isoparametric transformation to this reference elementary submesh, see Fig. 1. Then, the interpolation functions $N_{i}$ are associated with the nodes of the elementary submesh discretizing $\Omega_{k}$. The nodes of the elementary submesh lying on the boundary of $\Omega_{k}$ are not introduced in the definition of $V_{k}$ because the functions of $V_{k}$ must vanish on the boundary.

The assembly of all the elementary submesh builds up a refined mesh discretizing the whole domain $\Omega$. The refined mesh could be used in the computation of a more accurate reference solution $u_{r}$. However, the computation of the reference solution as a global refined problem must be avoided because of the large amount of degrees of freedom involved.

The reference solution $u_{r}$ is associated with a reference error, $e_{r}:=u_{r}-u_{h}$. The reference error $e$, can be seen as the projection of the actual error $e$ on the interpolation space $W$ generated by the global refined mesh. The space $W$ includes the space $V\left(V:=V_{1} \oplus V_{2} \oplus \cdots \oplus V_{m}\right)$ because $W$ contains the functions $N_{i}$ generating each $V_{k}$, associated with the interior nodes, but also the interpolation functions associated with the nodes lying on the

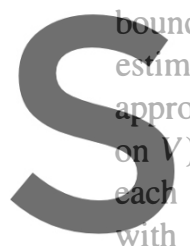
oundary of each element estimate given by $\varepsilon$ also W) is already an initial estir subspace $V_{k}$. Each projecti

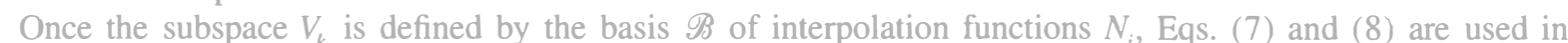

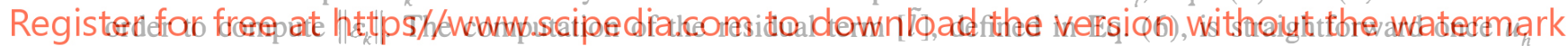
belongs to the reference space $W$. Then, $u_{h}$ can be expressed by the column vector $\left[u_{h}\right]_{\square}$ of its nodal values in

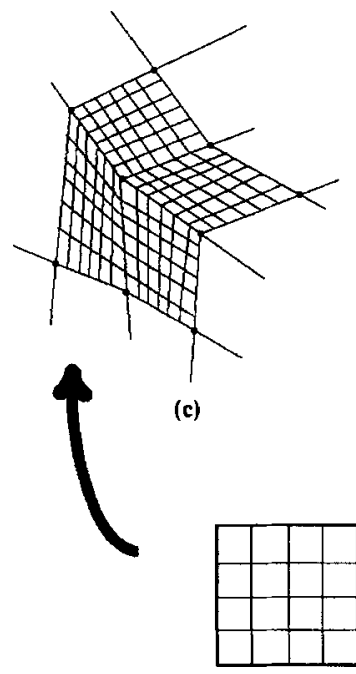

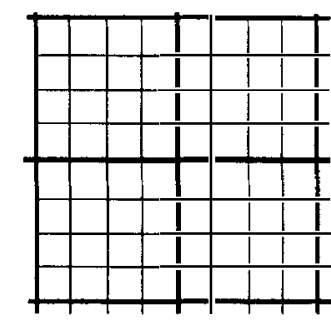

(b)

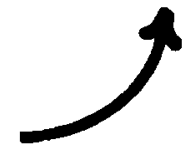

(a)

Fig. 1. Reference elementary submesh (a) and induced elementary submeshes over regular (b) and arbitrary (c) meshes. 
the elementary submesh discretizing $\Omega_{k}$. Thus, $a\left(u_{h}, N_{j}\right)$, see right-hand side of Eq. (6), is easily computed from the product of $\boldsymbol{K}$ and $\left[u_{h}\right]_{\mathscr{B}}$. Finally, we get

$$
[\tilde{l}]=[l]-K\left[u_{h}\right]_{\mathscr{B}} .
$$

This expression determines the right-hand side of Eq. (7) as a function of the terms appearing in the usual FE approach of a problem. The residual, i.e. the right-hand side of Eq. (7), does not need integration of the approximate solution, because integrals have been carried out when $\boldsymbol{K}$ was generated.

The residual term $[\tilde{l}]$ associated with a subspace $V_{k}$ with functions defined over an element $\Omega_{k}$ is called interior residual. The estimator given by Eq. (7) with this definition of the $V_{k}$ is called interior estimator. The interior estimator is characterized by the choice of the elementary submeshes.

It is very simple to define elementary submeshes for quadrilateral elements using a structured regular mesh of the reference square, see Fig. 1. It is important to notice that although the elementary submesh is chosen structured, the original computational mesh can be unstructured. These kind of submeshes verify that:

(a) they are simply defined on the reference element,

(b) they can represent the restriction of $u_{h}$ to $\Omega_{k}$ and

(c) they can be uniformly refined as far as the user wishes.

Computing the projection $\varepsilon_{k}$ as described in Eq. (7) is equivalent to solving a local problem in $\Omega_{k}$, with $\Omega_{k}$ discretized by the elementary submesh and where homogeneous Diruchlet boundary conditions are imposed on the boundary of $\Omega_{k}$.

The norm of $\varepsilon_{k}$ increases with the 'size' of the associated subspace $V_{k}$ and the number of degrees of freedom of the elementary submesh. A sequence of approximations based on refined submeshes has monotonic growth on norms and it is bounded by the norm of the exact and the reference error. Therefore, as the submesh is refined, the norm of the projections (local and global) converges to a value which is an underestimation of the error. This underestimation forcing the approximate functio Many authors [11-13] solve estimators is to use the
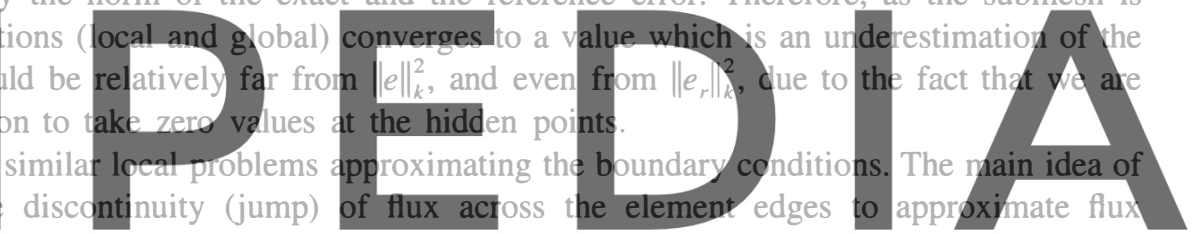

(Neumann) boundary conditions for the error equation, on the boundary of each $\Omega_{\mathrm{k}}$. Different authors use

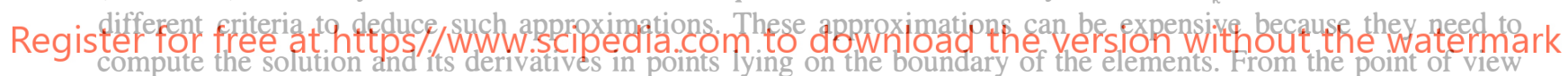

of the present projection approach, it is possible to take into account the information of flux jumps across edges by computing projections on subspaces of functions defined on subdomains including the edges. In the next section we introduce a new family of projections based on a new partition of $\Omega$. Finally, we compute a new error estimate combining the projections on the two families. Some compatibility conditions are imposed in order to make this combination possible and to still get a lower bound.

\section{Enrichment of the estimation. Overlapped meshing}

Up to now, we have computed local projections $\varepsilon_{k}$ of the error $e$ on subspaces $V_{k}$. We have used a partition of $\Omega$ in subdomains $\Omega_{k}\left(k=1, \ldots, m\right.$, namely the elements). Each subspace $V_{k}$ was defined by a submesh over the subdomain $\Omega_{k}$. The norms of $\varepsilon_{k}$ were taken as approximations of local measures of the true error $e$. Local and global approximations, $\varepsilon_{k}$ and $\varepsilon$, given by (8), (11) and (12), are lower bounds of the exact and reference error norms. We realized that this approximation could be poor. We would like to add new terms to the error estimation (9), in order to get closer to the exact error norm and still keep the lower bounding.

Since $\varepsilon$ is the projection of the reference error $e_{r}$ on the space $V, e$ can be uniquely expressed as the sum of $\varepsilon$ and a function $e_{r}^{\perp}$, orthogonal to $\varepsilon$, such that $e_{r}=\varepsilon+e_{r}^{\perp}$. Using the Pythagoras theorem we can write $\|\varepsilon\|^{2}=\left\|e_{r}\right\|^{2}-\left\|e_{r}^{\perp}\right\|^{2}$, therefore the norm of $e_{r}^{\perp}$ is the underestimation of the global reference error by the global interior estimate. The goal of the enrichment of the estimation is to approximate the forgotten part of the reference error $e_{r}^{\perp}$ and to add it to the first (interior) estimates. The function $e_{r}^{\perp}$ belongs to $V^{\perp}$, the orthogonal space of $V, V:=V_{1} \oplus V_{2} \oplus \cdots \oplus V_{m}$, in the reference interpolation space $W$. As we want to approximate $e_{r}^{\perp}$ by a projection, we would like to project on a subspace included in $V^{\perp}$. 
Let us consider a new partition of $\Omega$ into subdomains $\Lambda_{l}\left(l=1, \ldots, m^{\prime}\right)$. In order to make clear the difference between the two partitions, subdomains denoted by $\Lambda_{l}$ are called patches, while $\Omega_{k}$ are the actual elements. Every patch $\Lambda_{l}$ is discretized by a patch-submesh generating a subspace $U_{l}$ in $H_{0}^{1}\left(\Lambda_{l}\right)$. The subspaces $U_{l}$ are generated in the same way as the spaces $V_{k}$ associated with the elements. In order to approximate the reference error, the elements of each patch-submesh are chosen to be elements of elementary submeshes and, then also elements of the refined global mesh generating $W$. Patch-submeshes and elementary-submeshes share nodes and elements.

Since subdomains $\Lambda_{l}$ have zero measure intersections, subspaces $U_{l}$ are mutually orthogonal. The projections of $e_{r}$ (or $e$ ) on the $U_{l}$ 's can be thought of as a new family of local approximations to $e_{r}$ over different domains.

Let $\tilde{U}_{l}$ be the part of $U_{l}$ orthogonal to the interior global estimate $\varepsilon$. Since $\tilde{U}_{l}$ is a restriction of $U_{l}$ subject to one linear constraint, the dimension of the subspace $\tilde{U}_{l}$ is, at least, the dimension of $U_{l}$ minus one. Let $\eta_{l}$ denote the projection of the reference error $e_{r}$ on $\tilde{U}_{l}$.

Since $\varepsilon$ and each $\eta_{l}$ are orthogonal Bessel's inequality applies again,

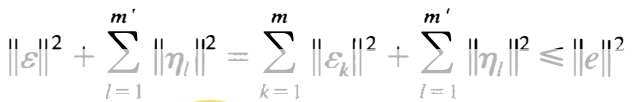

The orthogonality to $\varepsilon$ does not impose $\tilde{U}_{l}$ to be included in $V^{\perp}$. Actually, a restriction of $U_{l}$ ensuring orthogonality to the space $V$ would reduce it to the null subspace. This is due to the fact that, in general, the dimension of the subspace $U_{l}$ is less than the total number of nodes which are in the elements intersecting the corresponding $\Lambda_{l}$. Since we are obtaining a lower bound of the error, we prefer to impose the minimal restriction allowing to add the new contributions to the interior estimates as is done in Eq. (14).
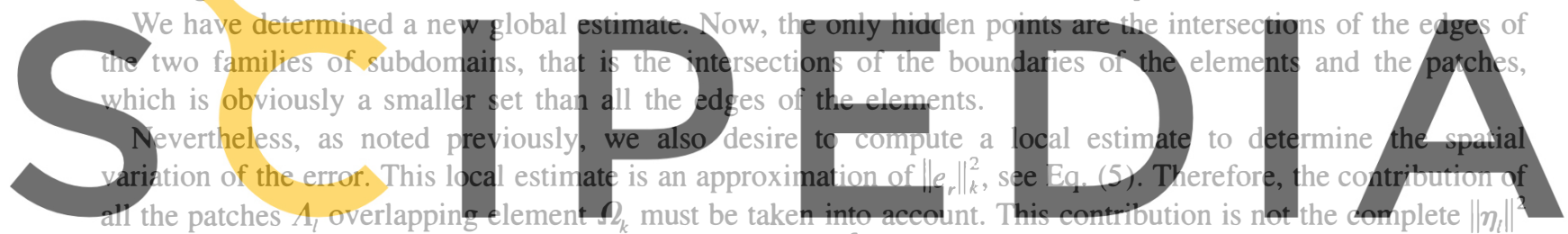

but the local norm restricted to $\Omega_{k}$, namely $a_{k}\left(\eta_{l}, \eta_{l}\right)=\left\|\eta_{l}\right\|_{k}^{2}$. Recall that $\eta_{l}$ has support on $\Lambda_{l}$, therefore it is zero

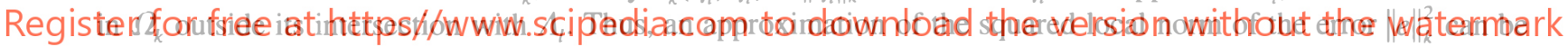
evaluated using

$$
\left\|\varepsilon_{k}\right\|_{k}^{2}+\sum_{l}\left\|\eta_{l}\right\|_{k}^{2}
$$

where index $l$ in the summation takes the values such that the patch $\Lambda_{l}$ overlaps the element $\Omega_{k}$.

Notice that the local norm of $\eta_{l}$ (restricted to $\Lambda_{l}$ ) is easily computed using an expression similar to (8). However, the computation of its contribution to element $\Omega_{k}$, i.e. $\left\|\eta_{l}\right\|_{k}$, needs to identify the elements of the patch-submeshes belonging to $\Omega_{k}$. This can be cumbersome. Therefore, in order to further simplify the computations another approximation is performed. We refer to equally distribute the squared norm of $\eta_{l},\left\|\eta_{l}\right\|^{2}$, over all the elements overlapping the patch $\Lambda_{l}$. That means we are approximating the quantities defined in (15) by

$$
\left\|\varepsilon_{k}\right\|_{k}^{2}+\sum_{l} \frac{\left\|\eta_{l}\right\|^{2}}{m_{l}}
$$

where $m_{l}$ is the number of elements overlapping the patch $\Lambda_{l}$ and index $l$ has the same range of variation as in Eq. (15). This approximation looses the lower bound property, in particular when elements with small error are next to elements with large error. However, the computational benefits are important and the examples in the next section show that the lower bound property is only lost in a negligible number of elements.

It is important to notice that the orthogonality condition to the interior global approximation $\varepsilon$ can be easily implemented if the nodes of the patch-submeshes (discretization of $\Lambda_{l}$ ) and the nodes of the elementarysubmeshes (discretization $\Omega_{k}$ ) are the same. Then, the selection of the subdomains $\Lambda_{l}$ is induced by the discretization of $\Omega_{k}$. We must choose at the same time the geometry and the meshing of $\Lambda_{l}$ conditioned to the 

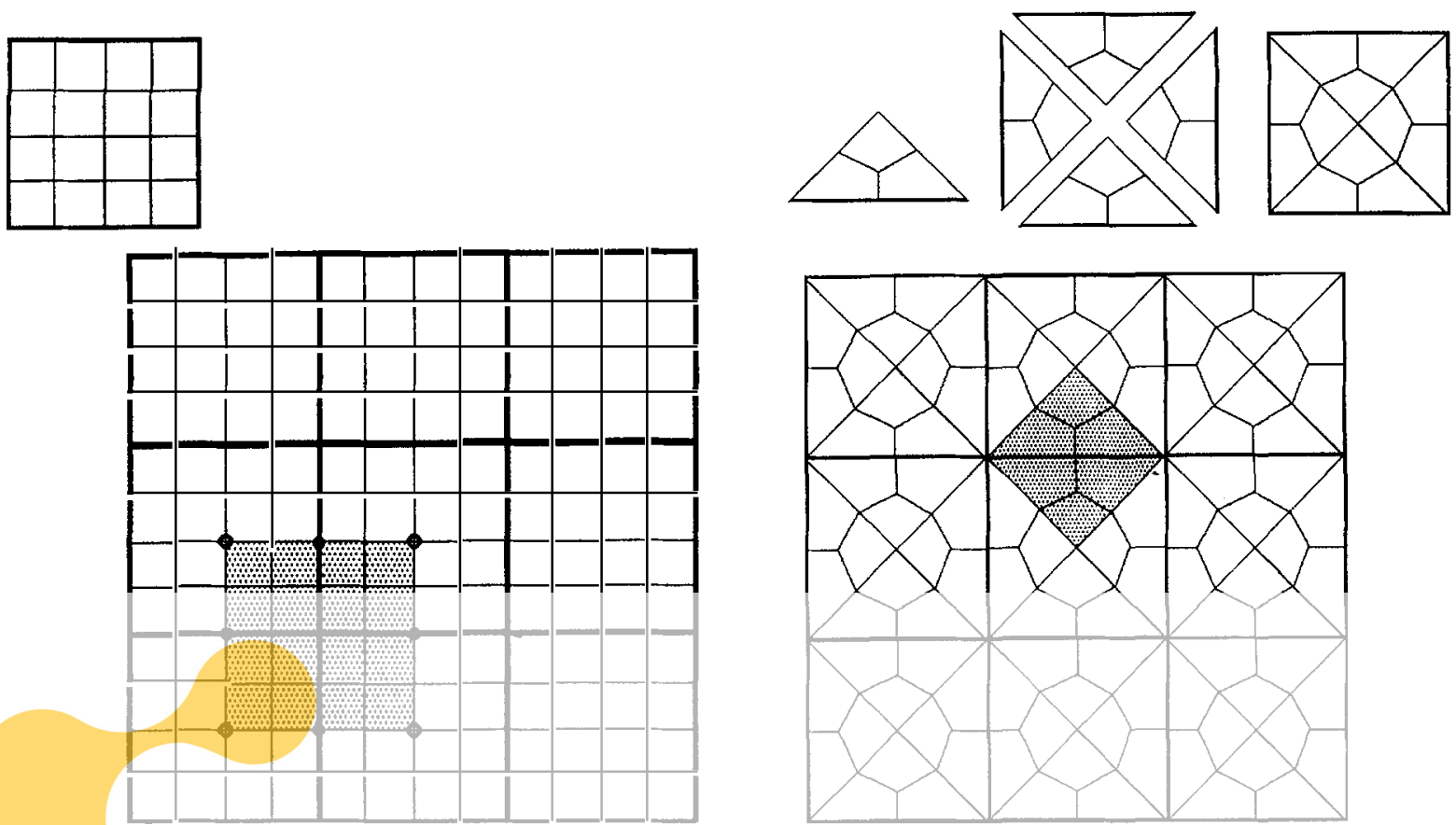

Fig. 2. Node patch submesh (shaded) associated with the regular

Fig. 3. Edge patch submesh (shaded) and elementary submeshes
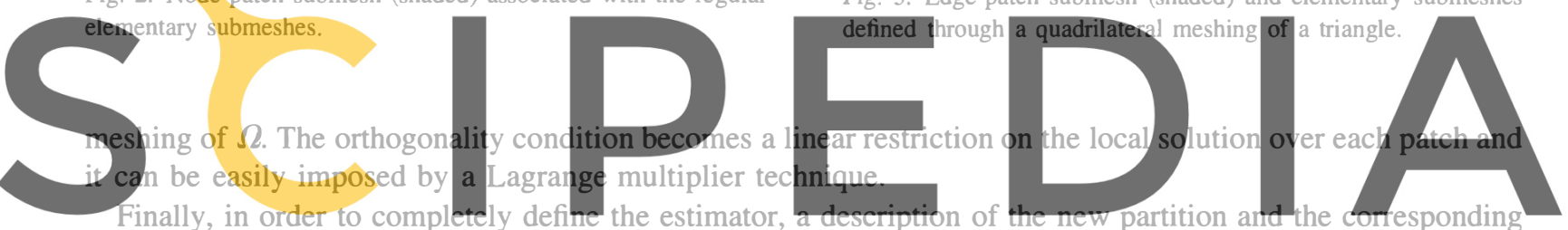

patch-submeshes is needed. If we consider an interior estimator as defined in Section 4, see Fig. 1, a set of

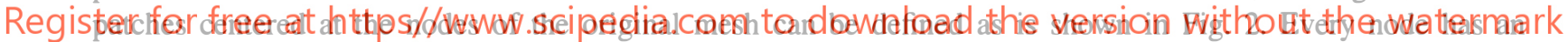

associated $\Lambda_{l}$ with vertices at the centers of adjacent elements and the middle points of the edges. The discretization of the patches is induced by the discretization of the elements. The hidden points of the total estimation are only the centers of the interior edges of elements. The estimator induced by this geometric definition is denoted by nodal-patch estimator.

It is possible to create an elementary-submesh and a patch definition ensuring that the hidden points of the total estimation are a priori chosen points, for instance the nodes of the original mesh. Fig. 3 shows how such submeshes and patches are defined. It is worth remarking that with this geometric definition, projection $\eta_{l}$ includes the effect of flux jump across the edge covered by the patch $\Lambda_{l}$. This estimator is denoted by edge-patch estimator.

In the definition of the estimator we have followed a particular projection strategy. In fact, we first obtain an interior projection $\varepsilon_{k}$ over each $V_{k}$ and then we use the global interior approximation $\varepsilon$ to impose a restriction for each $U_{l}$. The sum of the projections $\varepsilon_{k}$ and $\eta_{l}$ is only a lower bound of the full projection on a global space generated by both the two families $V_{k}$ 's and $U_{l}$ 's. The projection strategy could theoretically fail, depending both on the definition of the submeshes and the patches, see [15] for details. Nevertheless, in the numerical examples this pathology has never been noticed.

In the following sections we show some examples and we compare the difference of the nodal-patch estimates and the edge-patch estimates in a simple problem.

\section{Examples}

The practical analysis of an error estimator is based on the study of the effectivity index. The effectivity index is defined as the ratio of the measures of the actual and the estimated error. It can be defined either globally or 
locally. If the exact analytical solution of the problem is known, the effectivity index can be computed. If not, in order to study the performance of the estimator, the reference error $e_{r}$ can replace the exact error $e$ in the computation of the effectivity index. The lower bound property of the estimator is equivalent to have effectivity indexes lower than 1. The global effectivity index is lower than 1 even if it is computed using the reference solution (the estimate undervaluates the reference error). Therefore, local effectivity indexes are expected to be also lower than 1 .

We first present a simple unidimensional problem with known analytical solution. Let us consider the ordinary differential equation

$$
-\frac{\mathrm{d}^{2} u}{\mathrm{~d} x^{2}}(x)=6 x^{2}-3 x
$$

in $\Omega=$ ]0, 1[ with homogeneous Dirichlet conditions $u(0)=u(1)=0$. The exact solution of this problem is $u(x)=\left(x^{3}-x^{4}\right) / 2$. We solve the problem using the FE method with regular uniform meshes. We obtain two approximate solutions: $u_{h 1}$ with a mesh of 20 linear (two-nodes) elements and $u_{h 2}$ with a mesh of 15 quadratic (three-nodes) elements. The errors $e_{1}=u-u_{h 1}$ and $e_{2}=u-u_{h 2}$ are known, therefore we can compute local energy norms of the exact error on every element for both meshes. In the error estimation procedure, we first compute the projection of error on interior subspaces generated by uniform element submeshes. We compute projections for elementary submeshes defined by different numbers of subelements. Global results are shown in Tables 1 and 2. Increasing the number of degrees of freedom in the elementary submesh the global estimate converges very quickly to the global measure of exact error. The distributions of local error are shown in Fig. 4.

The estimates presented in Fig. 4 have been computed with coarse submeshes ( 5 linear elements and 2 quadratic elements), for finer submeshes the plots of exact and approximate error distributions cannot be distinguished,

\section{The estimated global error}

into account interior projections

that in such simple problerns,

approximation properties
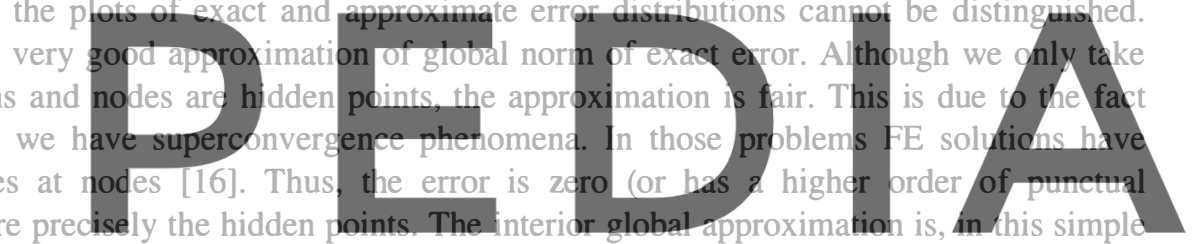

case, a good one. In other words, Dirichlet homogeneous conditions on local error equations are very accurate.

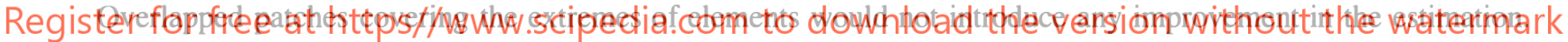

The second example is a plane Laplacian problem with a simple analytical solution. We consider the L-shaped

domain $\Omega$ of Fig. 5 and we state the Laplacian equation

$$
\frac{\partial^{2} u}{\partial x^{2}}+\frac{\partial^{2} u}{\partial y^{2}}=4 \text { in } \Omega \text {. }
$$

Table 1

Global error estimates and effectivity index for unidimensional problem with linear elements

\begin{tabular}{llll}
\hline $\begin{array}{l}\text { Element submesh } \\
\text { free nodes }\end{array}$ & $\begin{array}{l}\text { Element submesh } \\
\text { elements }\end{array}$ & Global error estimate & Global effectivity index \\
\hline 2 & 3 & 0.01488 & $94.3 \%$ \\
4 & 5 & 0.015463 & $98.0 \%$ \\
8 & 9 & 0.015687 & $99.4 \%$ \\
16 & 17 & 0.015758 & $99.8 \%$ \\
\hline
\end{tabular}

Table 2

Global error estimates and effectivity index for unidimensional problem with quadratic elements

\begin{tabular}{llll}
\hline $\begin{array}{l}\text { Element submesh } \\
\text { free nodes }\end{array}$ & $\begin{array}{l}\text { Element submesh } \\
\text { elements }\end{array}$ & Global error estimate & Global effectivity index \\
\hline 3 & 2 & $7.3403 \times 10^{-3}$ & $96.80 \%$ \\
5 & 3 & $7.5345 \times 10^{-3}$ & $99.40 \%$ \\
11 & 6 & $7.5799 \times 10^{3}$ & $99.98 \%$ \\
\hline
\end{tabular}



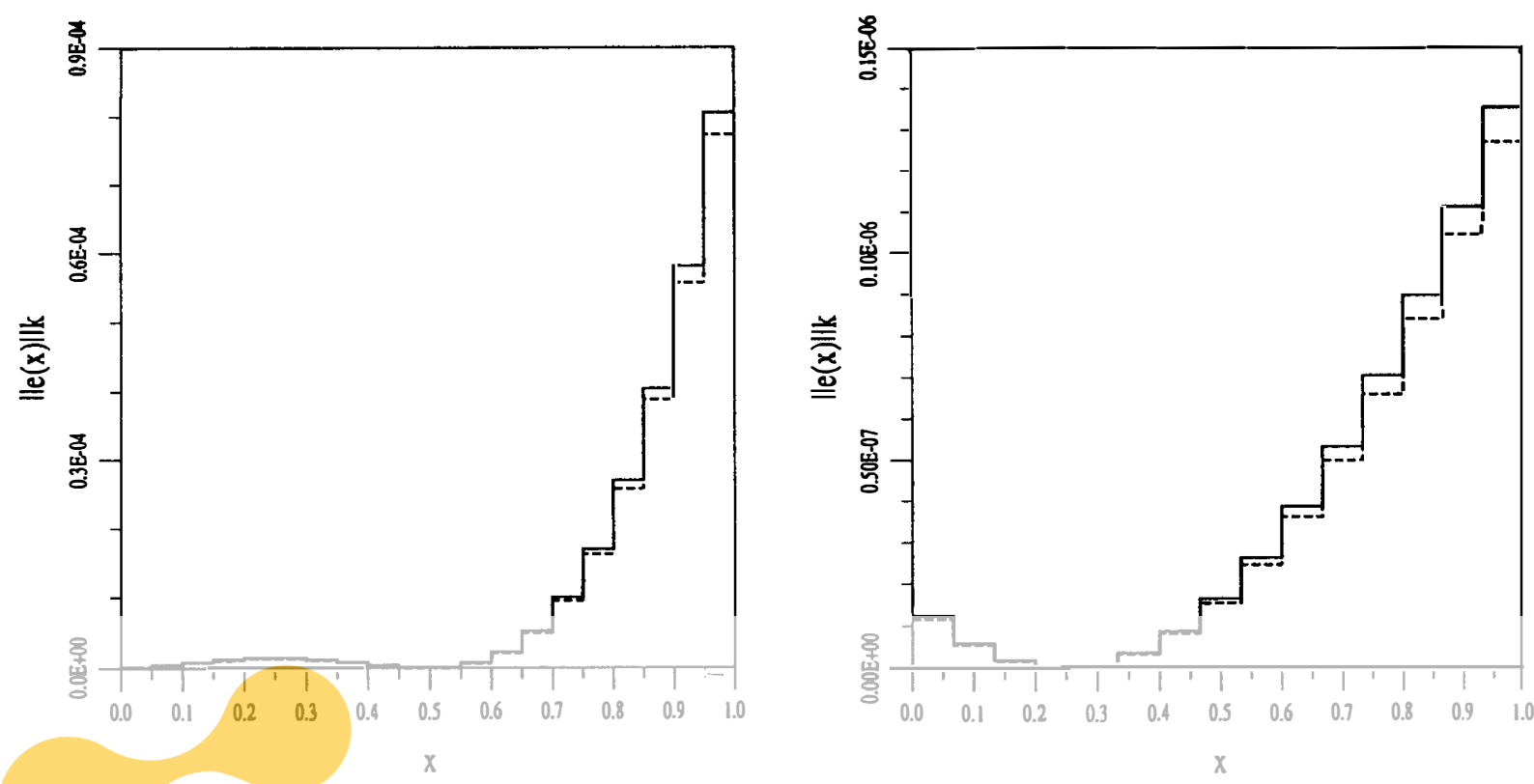

Fig. 4. Distribution of error measures, exact error (solid line) and estimated error (dashed line) for linear elements (left) and quadratic elements (right).

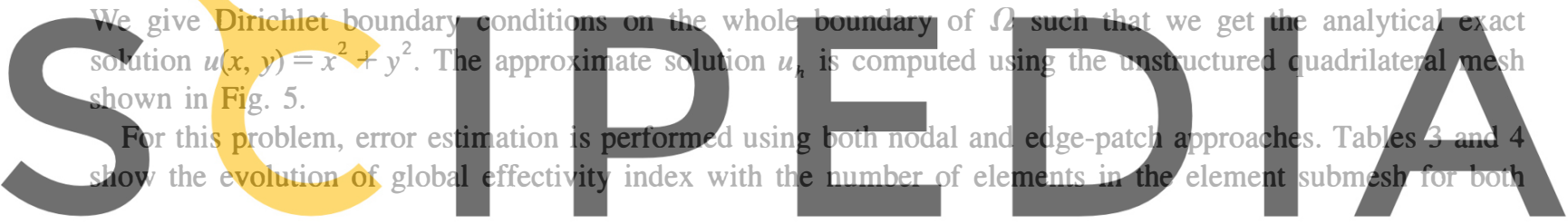

Register for free at https//www.scipedia.com to download the version without the watermark
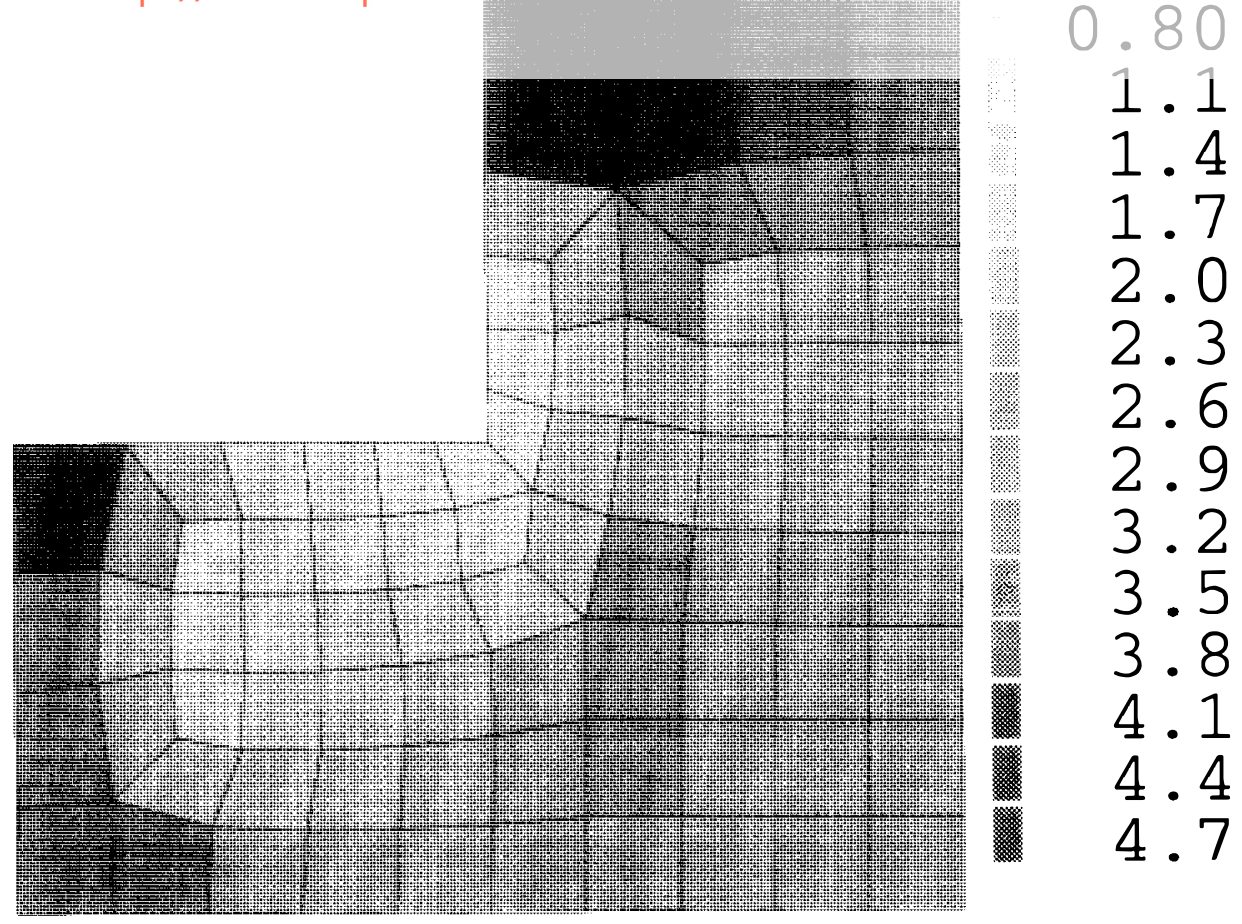

Fig. 5. Distribution of the exact error. 
Table 3

Global error estimates and effectivity index for bidimensional Laplacian problem with four noded quadrilateral elements and nodal patch submeshes

\begin{tabular}{rlll}
\hline $\begin{array}{l}\text { Element submesh } \\
\text { free nodes }\end{array}$ & $\begin{array}{l}\text { Element submesh } \\
\text { elements }\end{array}$ & Global error estimate & Global effectivity index \\
\hline 9 & 16 & 0.24759 & $91.9 \%$ \\
25 & 36 & 0.25435 & $94.4 \%$ \\
49 & 64 & 0.25670 & $95.3 \%$ \\
81 & 100 & 0.25780 & $95.7 \%$ \\
361 & 400 & 0.25934 & $97.5 \%$ \\
\hline
\end{tabular}

Table 4

Global error estimates and effectivity index for bidimensional Laplacian problem with four noded quadrilateral elements and side patch submeshes

\begin{tabular}{llll}
\hline $\begin{array}{l}\text { Element submesh } \\
\text { free nodes }\end{array}$ & $\begin{array}{l}\text { Element submesh } \\
\text { elements }\end{array}$ & Global error estimate & Global effectivity index \\
\hline 9 & 12 & 0.23381 & $86.8 \%$ \\
41 & 48 & 0.24315 & $90.2 \%$ \\
177 & 92 & 0.24691 & $91.6 \%$ \\
\hline
\end{tabular}

node-patch and edge-patch approaches. As we can see in Tables 3 and 4, in this case, the nodal-patch approach presents slightly better results. Anyway, in both cases the estimation fits the shape of the distribution of the local error. In fact, Fig. 5 shows the exact error distribution over the domain. Notice the similarity with Fig. 6 where the estimated error is presented. The represented estimation corresponds to a nodal-patch approach with elementary submeshes of 16 elements. As a measure of the quality of the estimator we also look at local
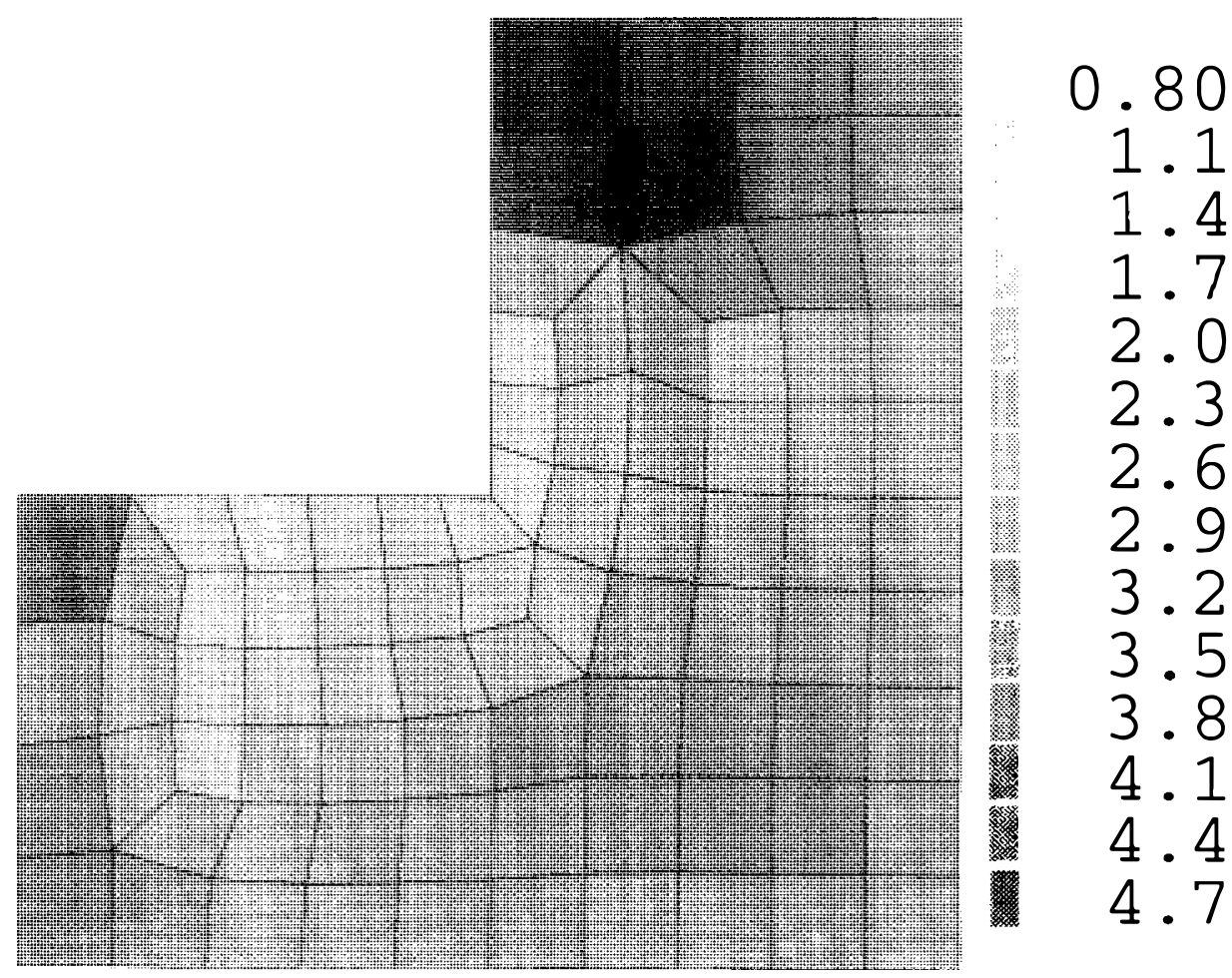

Fig. 6. Distribution of the estimated error. 


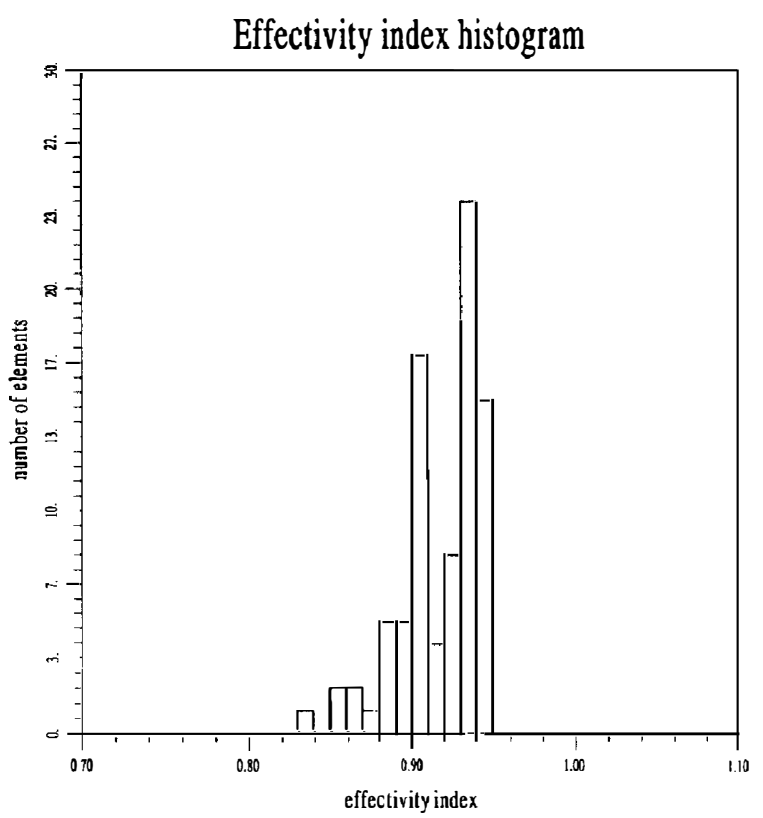

Fig. 7. Histogram representing the occurrences of the values of local effectivity index for the Laplacian problem (represented for the nodal patch estimator and the elementary submesh of 16 elements).

effectivity indexes. The histogram representing the occurrences of the values of local effectivity index (Fig. 7) shows the distribution of local underestimation and demonstrates that the spatial distribution of the local effectivity index is quite uniform (the values are concentrated between 0.83 and 0.95 ). So, the equality of the estimation is roughly the same in the whole domain. This is very important if the estimator is used in an

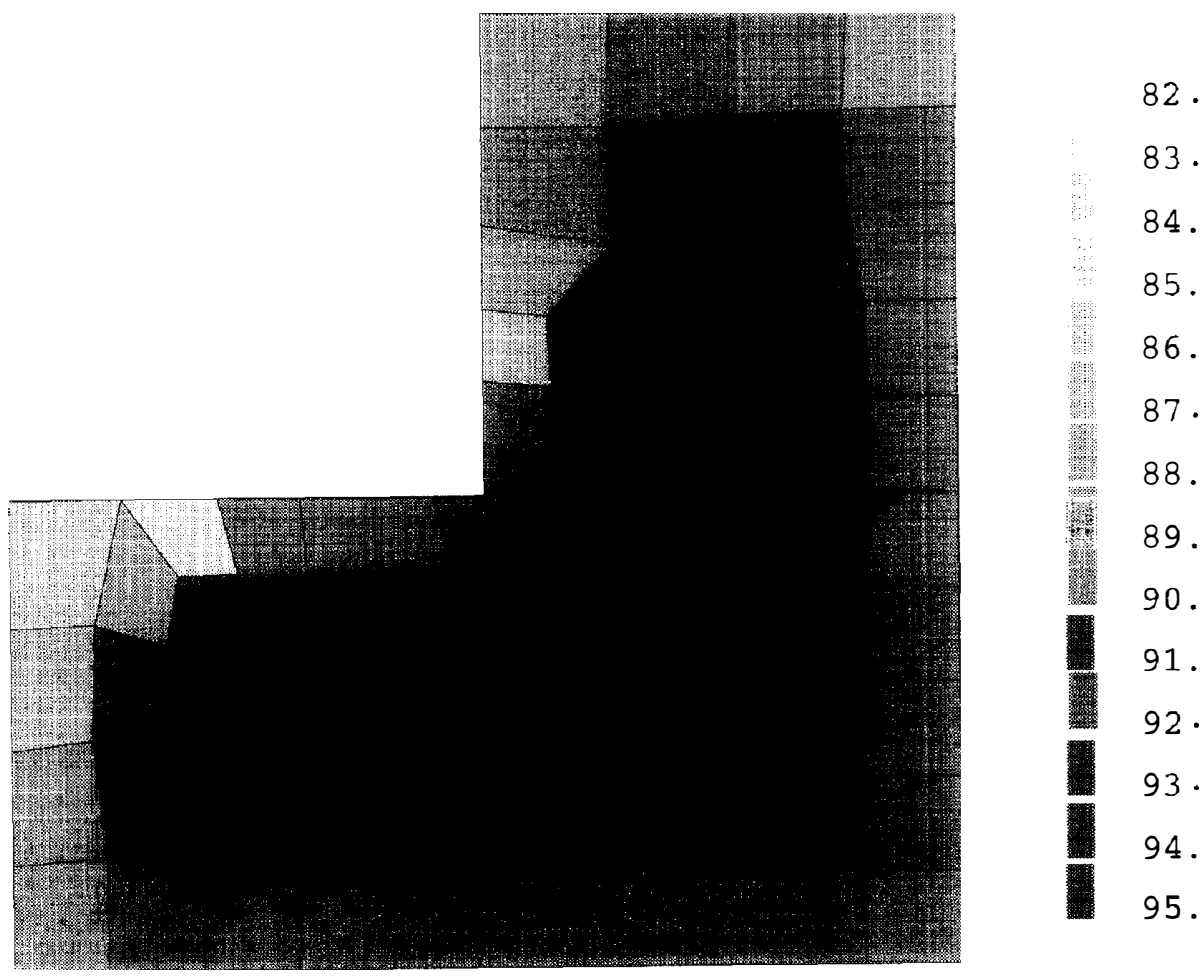

Fig. 8. Distribution of the local effectivity index. 


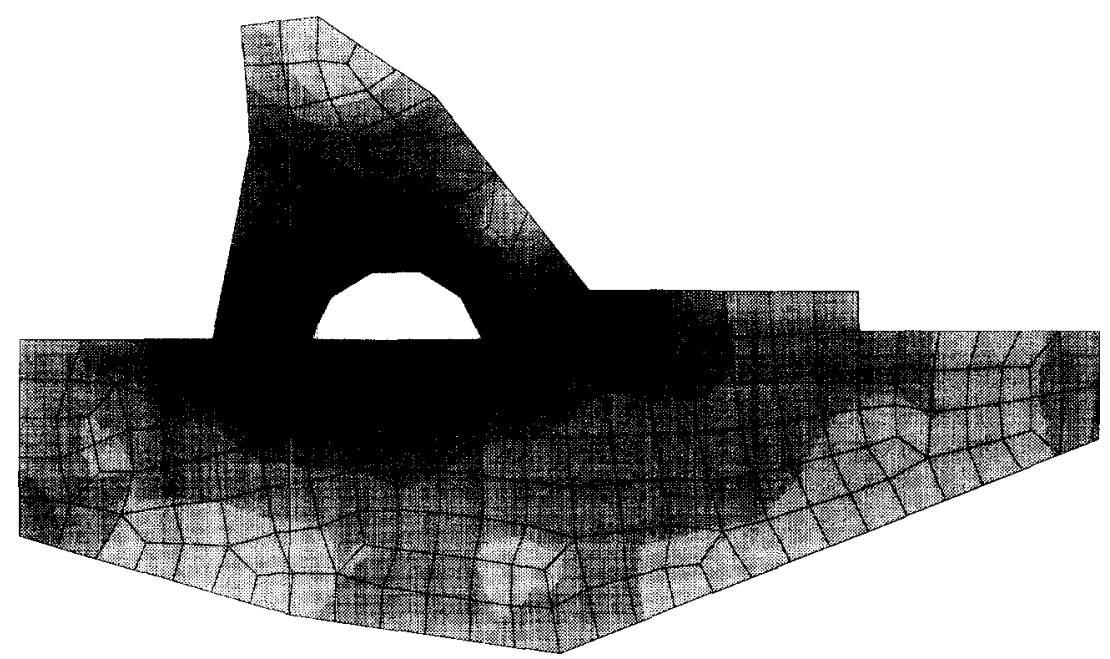

Fig. 9. Distribution of the reference error.

adaptive procedure because the mesh optimality criteria are based on the uniform spatial distribution of the error. Fig. 8 shows the spatial distribution of the effectivity index. Notice that, although the estimation is more accurate in the interior of the domain, on the boundary the effectivity index is over 0.92 .

This example shows that the estimator has a good behavior even in the elements near the Dirichlet boundary. Usually, standard error estimators present problems near the boundaries [2].

The third example is a plane elastic mechanical problem (in plane strain hypothesis). Displacements of a gravity dam under water pressure and concrete and rock weight loads are computed using FE. We prescribe displacements along the boundary representing the edge of the concerned ground domain.

Reference error distribution and estimated error distribution are shown in Figs. 9 and 10. The error

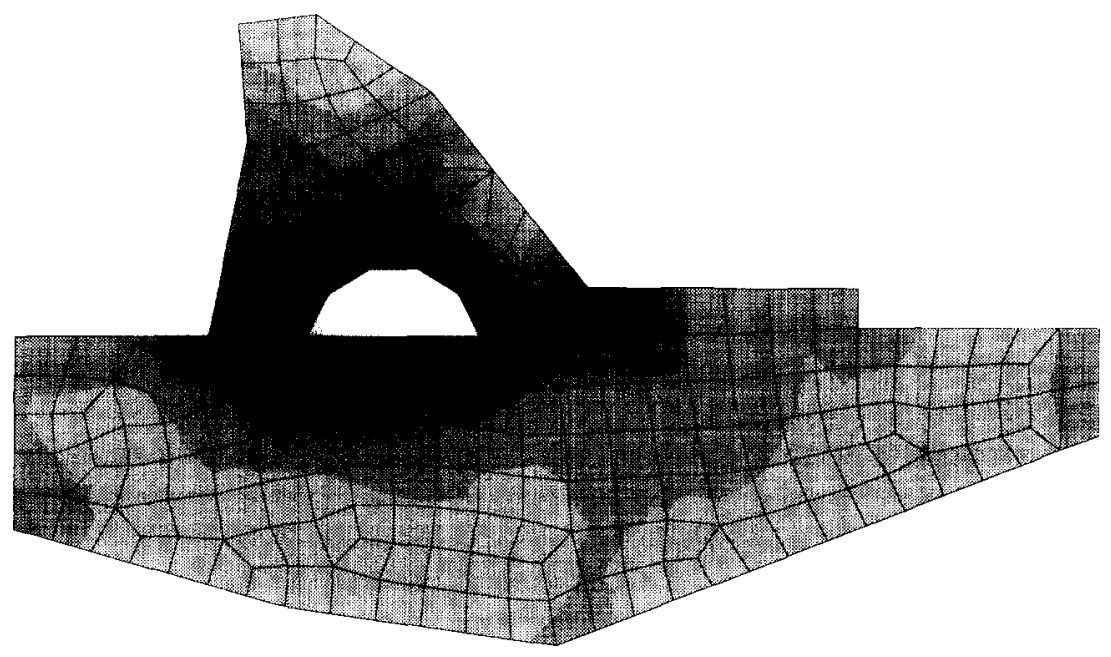

0.0

3.0

6.0

9.0

12 .

Fig. 10. Distribution of the estimated error. 


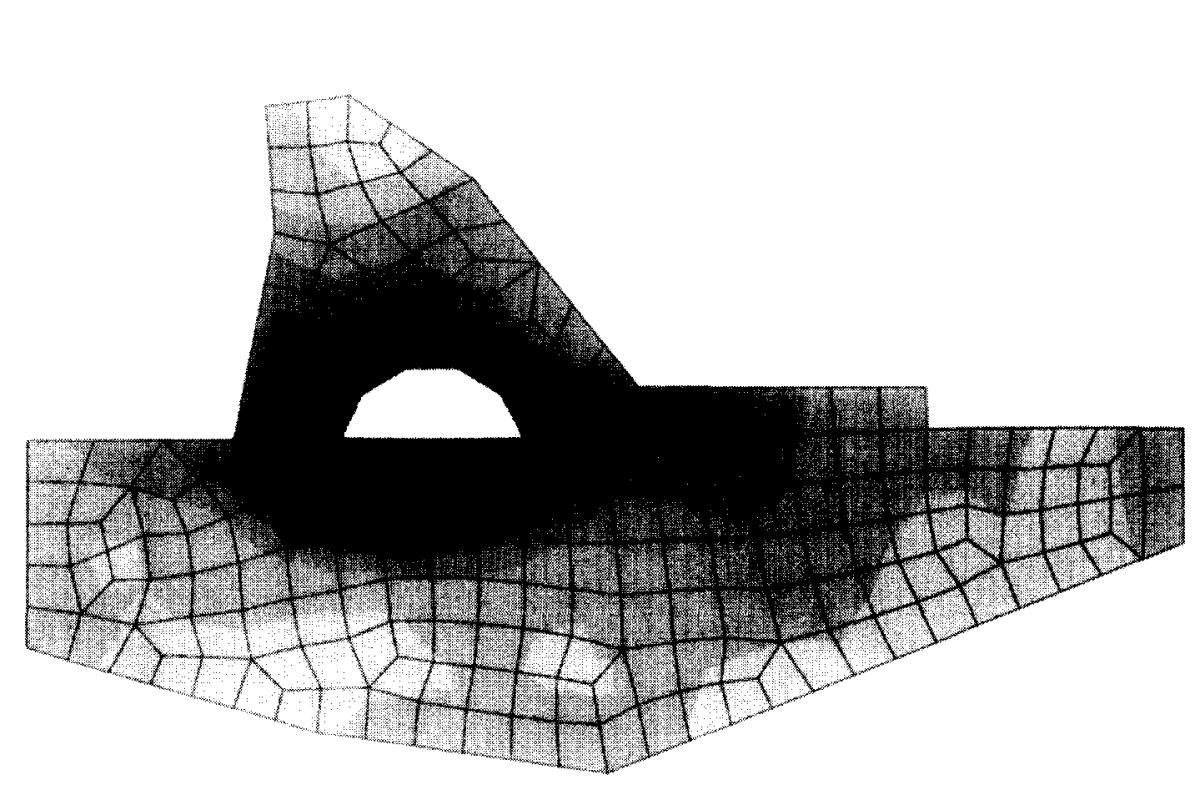

Fig. 11. Distribution of the Zienkiewicz Zhu estimated error.

distribution obtained with the Zienkiewicz-Zhu estimator described in [3] is shown in Fig. 11. Fig. 12 shows histograms of local values of effectivity index for the presented estimator and for the Zienkiewicz-Zhu estimator described in [3]. The values of the effectivity index associated with the presented estimator are concentrated in a narrower band. A uniform bias and a little dispersion in this histogram means that the quality of the estimation is uniform and, therefore, the estimator is well suited to an adaptive procedure. Figs. 13 and 14 represent the distributions of the local effectivity index for the presented estimator and the Zienkiewicz-Zhu estimator, respectively. Notice that spatial distribution of the local effectivity index for the presented estimator is more uniform.

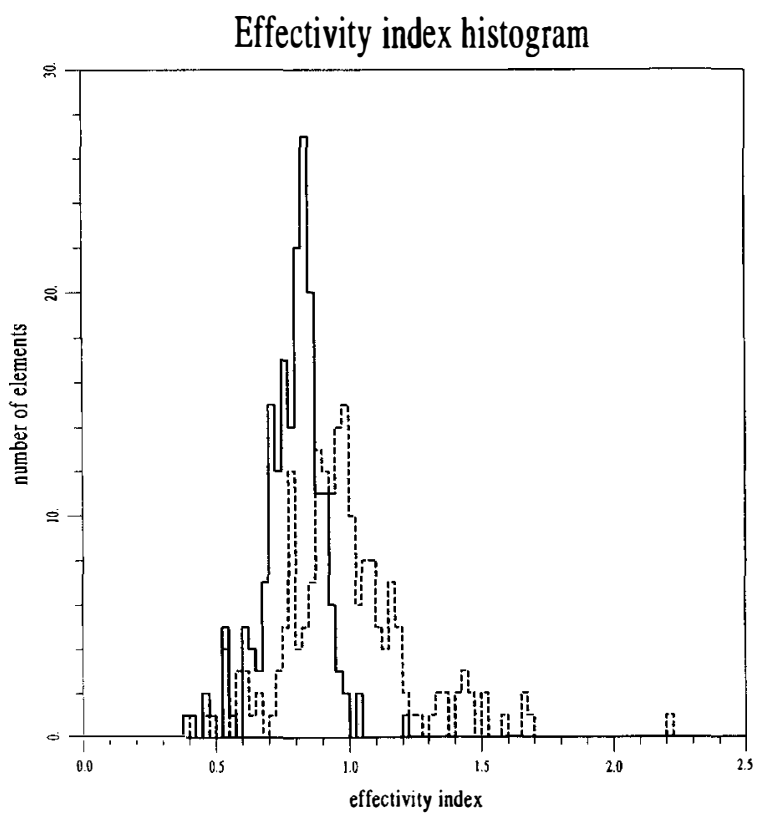

Fig. 12. Histogram representing the occurrences of the values of local effectivity index for the mechanical problem for both the presented estimator (solid line) and the Zienkiewicz Zhu estimator described in [3] (dashed line). 


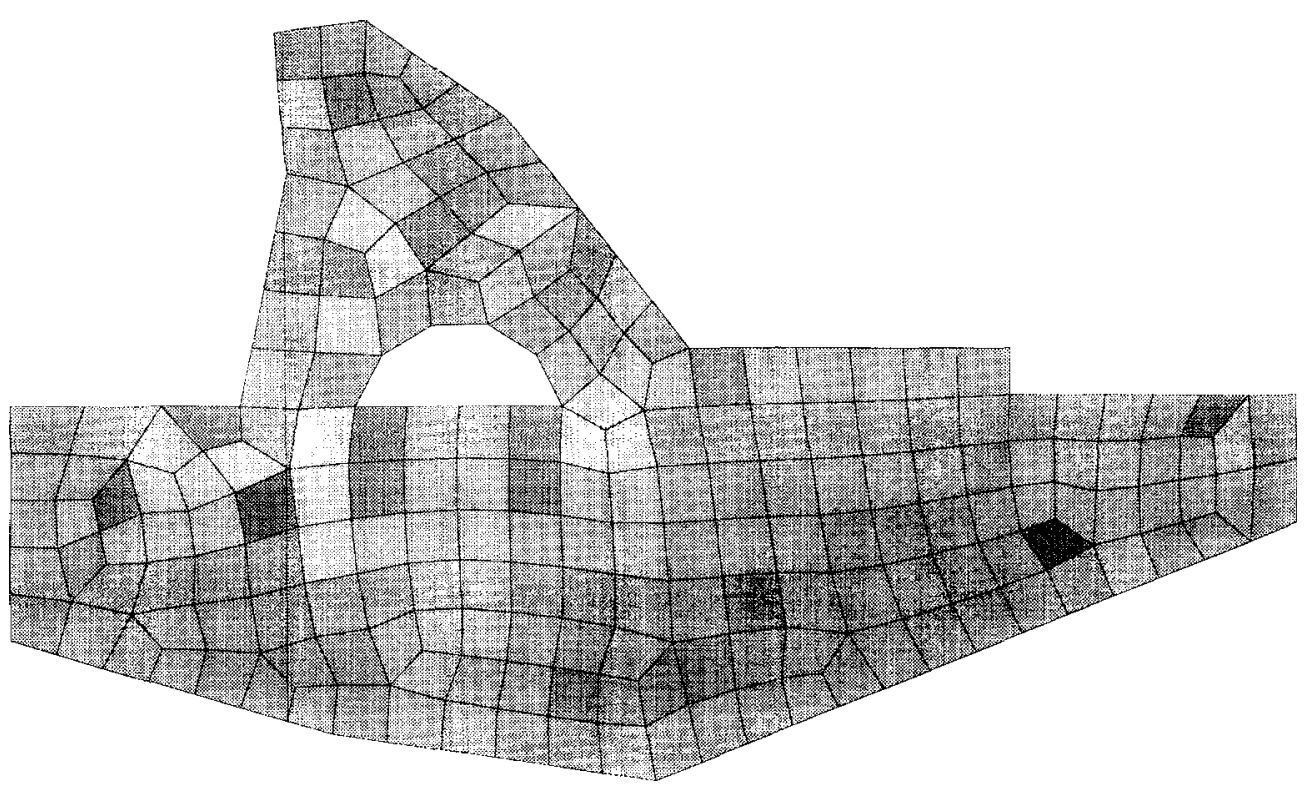

Fig. 13. Distribution of the local effectivity index for the presented estimator.

In this example, error estimation is used in an adaptive procedure. The objective is to obtain a solution with an error in energy norm lower than the 5\% of the global norm of the solution. Fig. 15 shows the sequence of meshes created using the error estimation. We followed the remeshing criterion presented in [17] and the mesh generator described in [18] in order to obtain new meshes given an equally distributed specific error. After the first step, the resulting mesh gives a solution with an error of $6.5 \%$ of the solution. The goal is attained in only two steps, the third mesh verifies the error prescription.

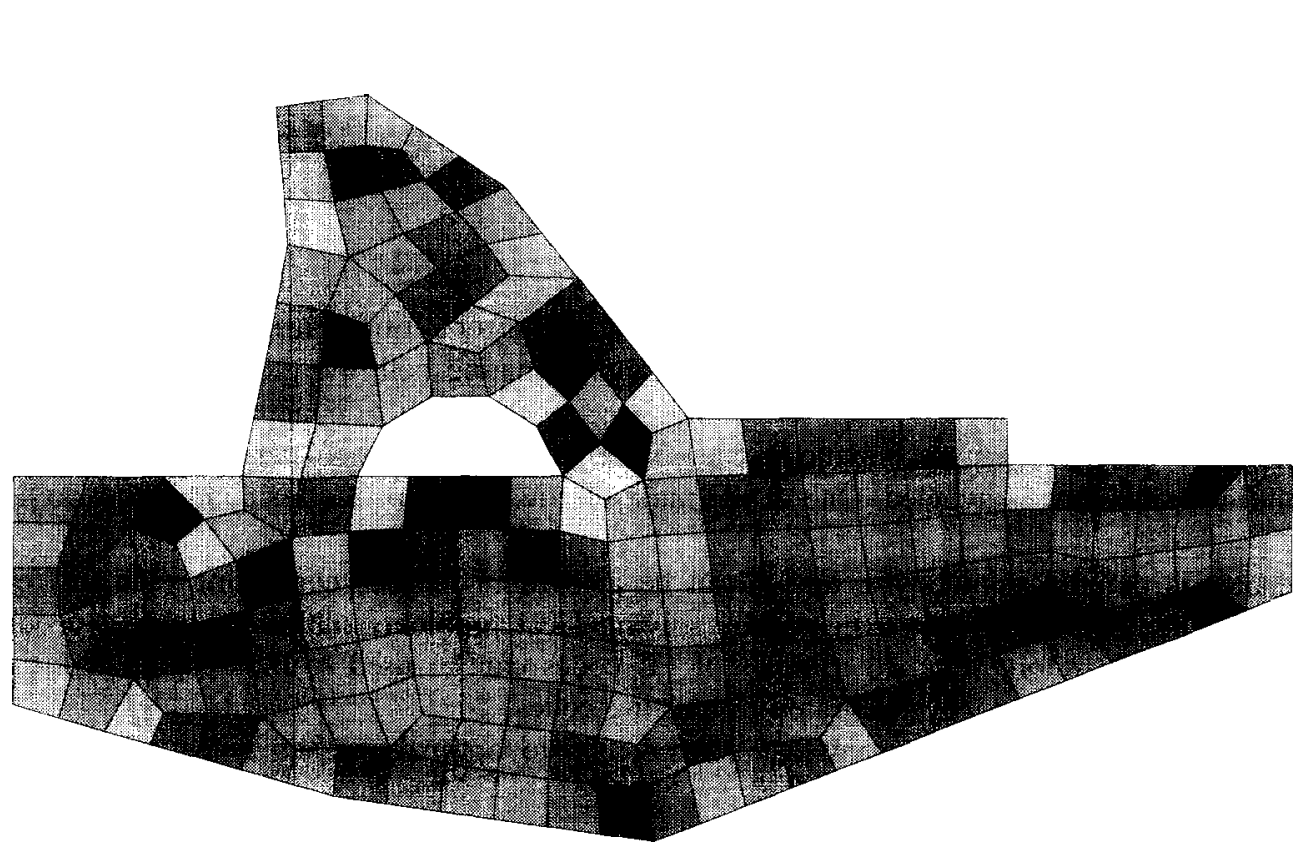

20.

30 .

40 .

50 .

60.

70.

80.

90 .

100.

110.

120.

130.

140.

Fig. 14. Distribution of the local effectivity index for Zienkiewicz Zhu estimator described in [3] 

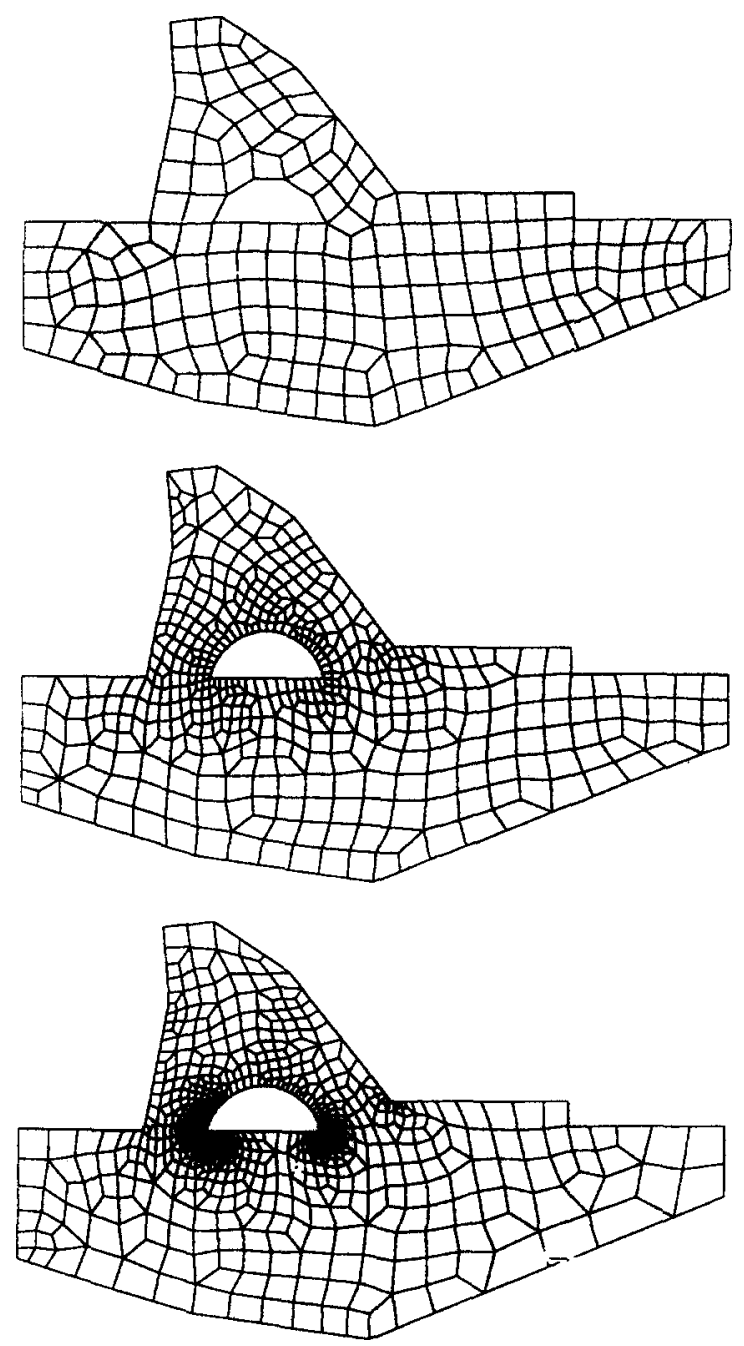

Fig. 15. Sequence of refined meshes leading to the optimal mesh giving $5 \%$ of relative error.

\section{Conclusions}

The methodology introduced in this paper provides a new approach to error estimation in $\mathrm{FE}$ analysis.

The presented lower bound estimator has a good performance approximating both the global value and the shape of the error. Although it is a residual estimator, it does not need to approximate boundary conditions for local problems. Therefore it is not necessary to compute flux jump cross the edges of each element and the error introduced by flux splitting techniques is avoided. One of the main disadvantages of the residual estimators is then precluded.

Moreover, the estimator is defined elementwise and it can be applied in meshes combining elements of different shapes. The reference solution is approximated by solving local problems subject to homogeneous Dirichlet boundary conditions. Therefore standard routines of FE codes can be used to build up each local problem. Consequently, the estimator is easily integrated in existing codes.

Adaptive procedures using this estimator present fast convergence to optimal meshes given a specified relative error.

Further studies about the quality and the robustness of the estimator under strong regularity conditions can be performed following the method described in [2] and [5]. 
Following the same idea, different estimates could be found by defining the local interpolation subspaces by higher order polynomial functions. The reference solution would be then a $p$-refined one and the orthogonality conditions should be imposed in a different way.

\section{References}

[1] J.Z. Zhu, E. Hinton and O.C. Zienkiewicz, Mesh enrichment again mesh regeneration using quadrilateral elements, Comm. Numer. Methods Engrg. 9 (1993) 547554.

[2] I. Babuška, T. Strouboulis and C.S. Upadhyay, A model study of a posteriori error estimators for linear elliptic problems. Error estimation in the interior of patchwise uniform grids of triangles, Comput. Methods Appl. Mech. Engrg. 114 (1994) 307378.

[3] O.C. Zienkiewicz and J.Z. Zhu, A simple error estimator and adaptive procedure for practical engineering analysis, Int. J. Numer. Methods Engrg. 24 (1987) 337357.

[4] O.C. Zienkiewicz and J.Z. Zhu, The superconvergent patch recovery (SPR) and adaptive finite element refinement, Comput. Methods Appl. Mech. Engrg. 101 (1992) 207224.

[5] I. Babuška, T. Strouboulis, C.S. Upadhyay, S.K. Gangaraj and K. Copps, Validation of a posteriori error estimators by numerical approach, Int. J. Numer. Methods Engrg. 37 (1994) 10731123.

[6] M.F. Wheeler and J.R. Whiteman, Superconvergent recovery of gradients on subdomains from piecewise linear finite element approximations, Numer. Methods Partial Diff. Eqns. 3 (1987) 357374.

[7] I. Babuška and C. Rheinboldt, A posteriori error estimates for the finite element method, Int. J. Numer. Methods Engrg. 12 (1978) 15971615.

[8] P. Ladevèze, J. P. Pelle and Ph. Rougeot, Error estimation and mesh optimization for classical finite elements, Engrg. Comput. 8 (1991) 6980.

[9] H. Ohtsubo and M. Kitamura, Numerical investigation of element wise a posteriori error estimation in two and three dimensional elastic problems, Int. J. Numer. Methods Engrg. 34 (1992) 969977.

[10] K. Erikkson and C. Johnson, Adaptive finite element methods for parabolic problems I: A linear model problem, SIAM J. Numer. Anal. 28 (1991) 4377.

[11] M. Ainsworth and J.T. Oden, A procedure for a posteriori error estimation for $h$-p finite element methods, Comput. Methods Appl. Mech. Engrg. 101 (1992) 7396.

[12] R.E. Bank and A. Weiser, Some a posteriori error estimators for elliptic partial differential equations, Math. Comput. 44 (1985) 283301.

[13] J.T. Oden, L. Demkowicz, W. Rachowicz and T.A. Westermann, Toward a universal $h-p$ adaptive finite element strategy, Part 2. A posteriori error estimation, Comput. Methods Appl. Mech. Engrg. 77 (1989) 113180.

[14] B. Tie and D. Aubry, Error estimates, $h$ adaptive strategy and hierarchical concept for non linear finite element method, in: C. Hirsch et al., eds., Proc. First European Conference on Numerical Methods in Engineering, Brussels (Elsevier, Amsterdam, 1992$) 113180$.

[15] P. Díez, Un nuevo estimador del error para el método de los elementos finitos, Doctoral Thesis, Universitat Politècnica de Catalunya, 1996.

[16] T.J.R. Hughes, The Finite Element Method (Prentice Hall, Englewood Cliffs, NJ, 1987).

[17] E. Oñate and G. Bugeda, A study of mesh optimization criteria in adaptive finite element analysis, Engrg. Comput. 10 (1993) 307321.

[18] J. Sarrate, M. Gutiérrez and A. Huerta, A new algorithm for unstructured quadrilateral mesh generation and rezoning, in: Ken Morgan et al., eds., Proc. VIII Int. Conf. on Finite Elements in Fluids, Barcelona (Pineridge Press, 1993) 745754. 